\title{
Estimación de los costes de publicación en revistas científicas indexadas en JCR en 2017 y 2018: el caso de la Psicología
}

\author{
María Peñaranda-Ortega*, Francisco González-Sala**, Julia Osca-Lluch *** \\ *Dpto. Psicología Básica y Metodología. Universidad de Murcia \\ Correo-e: mariap@um.es | ORCID iD: https://orcid.org/0000-0001-9916-0714 \\ ** Dpto. Psicología Evolutiva y de la Educación. Universidad de Valencia. \\ Correo-e: Francisco.Gonzalez-Sala@uv.es | ORCID iD: https://orcid.org/orcid.org/0000-0003-4124-7459 \\ $* * *$ Ingenio (CSIC-Universitat Politècnica de València). \\ Correo-e: m.julia.osca@uv.es | ORCID iD: https://orcid.org/orcid.org/0000-0002-0449-5878
}

Recibido: 10-07-20; $2^{a}$ versión: 23-09-20; Aceptado: 27-10-20; Publicado: 13-10-21

Cómo citar este artículo/Citation: Peñaranda-Ortega, M.; González-Sala, F.; Osca-Lluch, J. (2021). Estimación de los costes de publicación en revistas científicas indexadas en JCR en 2017 y 2018: el caso de la Psicología. Revista Española de Documentación Científica, 44 (4), e311. https://doi.org/ 10.3989/redc.2021. 4.1819.

Resumen: En el presente trabajo se evalúan los costes de publicación (Article Processing Charges) que se exigen por parte de las editoriales científicas, derivados de la publicación de artículos en sus revistas. Se han seleccionado las revistas incluidas en los cuartiles 1 y $2(\mathrm{Q} 1, \mathrm{Q} 2)$ en el Journal Citation Reports (JCR) para las once categorías en las que se subdivide Psychology, durante los años 2017 y 2018 . Se ha calculado el coste mayor o Golden OA y transformado en euros para todas las cantidades requeridas por parte de las editoriales. Para el total de 632 revistas analizadas en estos dos años, el coste final es de 135.514.886,39 $€$, de los cuales $67.027 .213,11 €$ pertenecen a las revistas del JCR 2017 y 68.487.673,28 € a JCR 2018. La media de coste de publicación de un trabajo fue de 2321,55 $€$. Son numerosas las conclusiones sobre la política científica y de publicación que los Estados deberían repensar.

Palabras clave: Revistas científicas; costes procesamiento artículos; Open Acess; editoriales científicos; Psicología.

\section{Estimate costs for publishing in JCR-indexed scientific journals in 2017 and 2018: the case of Psychology}

Abstract: This paper assesses the costs of publishing (Article Processing Charges) demanded by scientific publishers to publish articles in their journals. It have been selected the journals included in quartiles 1 and $2(\mathrm{Q} 1, \mathrm{Q} 2)$ in Journal Citation Reports (JCR) for the eleven categories in which Psychology is subdivided, during 2017 and 2018. The Golden OA has been calculated and converted into euros for all the amounts required by the publishers. As for the 362 journals which have been analysed within these two years and the total number of registers obtained was 632, the final cost is $€ 135,514,886.38, € 67,027,213.11$ of which relate to journals in JCR 2017, and $€ 68,487,673.28$ to JCR 2018. The average cost of publishing an article was $€ 2321.55$. There are statistically significant differences in the costs of publishing in open access according to the category of Psychology. There are many conclusions about scientific and publications policies which nations should rethink.

Keywords: scientific journals; article processing charges; Open Access; scientific editorials; Psychology.

Copyright: (c) 2021 CSIC. Este es un artículo de acceso abierto distribuido bajo los términos de la licencia de uso y distribución Creative Commons Reconocimiento 4.0 Internacional (CC BY 4.0). 


\section{INTRODUCCIÓN}

En la actualidad el análisis de la productividad científica ya es algo más que un interés de los responsables de la política científica y de los propios investigadores, es una necesidad que se relaciona con múltiples aspectos, como la financiación de las universidades y la evaluación y promoción de los investigadores. Es un hecho incuestionable que los trabajos publicados en revistas científicas con factor de impacto, constituyen un aspecto determinante para evaluar la ciencia en una gran cantidad de países, debido a sus políticas de financiación de la investigación. En el caso de España, esto sucede tanto a nivel nacional como autonómico (Musi-Lechuga y otros, 2005).

Las revistas constituyen, en numerosas disciplinas, el principal vehículo utilizado por los investigadores para dar a conocer sus trabajos. La publicación de los resultados alcanzados en las investigaciones es el último paso en el ciclo de la investigación científica $y$, las revistas científicas, desde su nacimiento a finales del siglo XVII, vienen cumpliendo algunas funciones básicas, como la periodicidad, alcanzándose la rapidez, concisión, especialización y novedad que, en la transmisión de conocimientos, demandaba la nueva sociedad científica (Delgado y Cordón, 1991).

La aparición, hace algunos años, de las primeras publicaciones periódicas electrónicas, que nacieron como complemento a la edición impresa, hizo suponer que en muy poco tiempo éste sería el medio por el que las comunidades científicas iban a difundir sus estudios e iban a estar informados de lo que se producía en las distintas disciplinas del conocimiento humano (Martin y Merlo, 2003). La mitad de las revistas españolas se publican en ambos formatos, cerca de un $30 \%$ publica solo en formato digital y un $20 \%$ lo hace en formato impreso (Claudio-González y otros, 2017). Sin embargo, con los avances en tecnologías de la información aplicadas al mundo de las revistas científicas, los sistemas de edición de revistas, inalterables durante más de trescientos años, se están viendo sacudidos por dos acontecimientos relacionados con la aparición de la edición electrónica a través de internet, como son la reducción de costes de producción y distribución y el aumento de la accesibilidad y visibilidad de los contenidos por medio del movimiento de acceso abierto (Barrueco, 2010).

La publicación de los resultados de la investigación en acceso abierto es la forma que llegó a ser consensuada para rendir cuentas a la sociedad sobre los recursos utilizados en la investigación, dado que gran parte de ésta se financia públicamente. El movimiento de acceso abierto emerge de la crisis generada por los costes de las publicaciones impresas en la década de los años 2000, y las oportunidades de acceso ofrecidas por la edición digital en la Web. Hoy en día está totalmente consolidado el acceso abierto para las revistas en formato digital. En este sentido, prácticamente la mitad de los artículos publicados en países de la Comunidad Europea, en Estados Unidos, Canadá, Japón y Brasil entre 2004 y 2011 estaban disponibles en abierto (Nassi-Calò, 2013). Además, con el paso del tiempo, se observa un incremento en el número de publicaciones que ofrecen la posibilidad de publicar en acceso abierto (Laakso y Björk 2012).

El acceso abierto se establece mediante múltiples vías o modos: ya sea a través de la modalidad conocida como "dorada" (Golden OA), que incluye las revistas que publican todos sus artículos en acceso abierto. Dentro de esta "vía dorada" también hay revistas que están subvencionadas al $100 \%$ por instituciones académicas, sociedades científicas, etc., y que no cobran por publicar (algunos llaman a esto "vía platino"), pero, en general, las revistas de acceso abierto requieren un pago por parte del autor (Lopez-Torres, 2015). Otra opción son las revistas híbridas, que siguen financiándose por las suscripciones pero ofrecen la llamada opción abierta (open choice), de manera que los autores pueden liberar sus artículos individuales pagando una cantidad, o bien en la modalidad conocida como "verde" (Green OA), que se produce con el archivo de artículos en repositorios institucionales o centrales posteriormente a su publicación en revistas (Claudio-González y otros, 2017).

En cualquier caso, la financiación es un gran condicionante en la investigación. Las revistas de acceso abierto necesitan una fuente de financiación y puede parecer obvio que la fuente deban ser los propios autores (Kaufman, 2014; López-Torres, 2015). Las fuentes más comunes de apoyo a la investigación incluyen a los propios investigadores y sus instituciones. Muchas revistas de la vía dorada cobran a los autores o a las instituciones a las que están afiliados, o bien a las agencias de financiación de proyectos, tasas de publicación conocidas como "tasa de procesamiento de artículo" (Article Processing Charge). Este sistema de pago por autor (author pay model o author side fee model) ha sido adoptado tanto por editoriales comerciales como por editoriales sin ánimo de lucro.

Pese a que actualmente los investigadores reciben poco dinero para financiar sus proyectos de investigación, este modelo de pagar por publicar detrae fondos de la investigación para poder publicar (López-Torres, 2015). Dallmeier-Thiessen y otros (2011) apuntan que en un $28 \%$ de los casos 
los fondos para publicar salen del dinero concedido a través de una subvención, en un $55 \%$ fueron fondos generales de subvenciones o del departamento, en un $12 \%$ de los propios investigadores y en un $5 \%$ otras fuentes. Solomon y Björk (2012) encuentran que en países desarrollados, el $30 \%$ de estos gastos son cubiertos por dinero de subvenciones, mientras que en países en vías de desarrollo el $39 \%$ de los gastos son cubiertos por los propios investigadores. Como consecuencia, los investigadores se ven inmersos en un entorno competitivo, abocados a adaptarse a un sistema de evaluación que implica necesariamente la asignación de fondos.

El importe de estas tasas, sin embargo, varía ampliamente entre los editores de las revistas comerciales y las asociaciones o sociedades científicas. Según Dewatripont y otros (2006); Bergstrom (2001) y Barschall (1988) los costes de publicación en revistas pertenecientes a editoriales comerciales eran mayores que aquellas publicadas por sociedades o asociaciones y universidades. Dewatripont y otros (2006) encuentran una relación positiva entre el número de citas y los cargos por publicar. Si bien suele haber un precio fijo por artículo publicado en cada revista, hay algunas editoriales que pueden ofrecer precios diferentes según el país de procedencia de los autores (Björk y Solomon, 2012). Existen-también diferencias entre áreas científicas, siendo las revistas biomédicas donde los cargos por publicar son mayores frente a aquellas revistas pertenecientes a las áreas sociales o humanidades (Björk y Solomon, 2015)

Una vertiente interesante que está surgiendo en los últimos años, consiste en relacionar la calidad científica de la investigación que se realiza en las universidades españolas con la financiación que éstas reciben de las distintas comunidades autónomas y, por tanto, del Estado, con el objetivo de verificar en qué medida se están aprovechando dichos recursos económicos (Buela-Casal y otros, 2012 , 2013), ya que, aproximadamente, el $80 \%$ de dicha actividad se financia a través de fondos públicos (Osuna, 2009). El éxito en la obtención de financiación para la investigación ha pasado, por tanto, a tener un gran peso en muchos sentidos, entre los cuales hay que mencionar la publicación de los resultados en revistas de impacto y, si es posible en abierto, con el fin de dar mayor visibilidad a los resultados, y así poder tener un mayor número de citas, ya que mediante el acceso abierto, los autores observan que sus artículos son más leídos y más citados. Son estos dos indicadores, la calidad de la publicación, principalmente en los dos primeros cuartiles del JCR (Journal Citation Reports) y el número de citas, aspectos muy valorados en Espa- ña, a través de los criterios establecidos por la Comisión Nacional Evaluadora de la Actividad Investigadora (CNEAI) (Ministerio de Ciencia, Innovación y Universidades, 2018), para la evaluación de las carreras académicas y científicas. Esto es lo que induce a los científicos a publicar necesariamente en estas publicaciones y a consultar esas fuentes, si bien, como apunta Wiley (2013), la calidad de la revista es uno de los primeros criterios que tienen en cuenta los investigadores a la hora de publicar sus trabajos. Su acceso, promoción y éxito en la carrera científica dependen inevitablemente de ello (Delgado, 2018a, 2018b).

Sin embargo, el coste elevado de publicar trabajos en algunas de las revistas, así como el efecto "cuello de botella" que se produce en ellas dada la enorme cantidad de trabajos que reciben, puede conducir a una desigualdad en cuanto a las posibilidades de publicación y, de tal modo, discriminar a investigadores o líneas de investigación que no atraigan el pertinente apoyo financiero, así como a jóvenes investigadores que carecen de los medios para pagar por la publicación (Swan y Brown, 2004). También puede darse el caso de que la producción científica de algunas áreas o disciplinas pueda disminuir como consecuencia de las limitaciones económicas (Hernández Borges y otros, 2006).

Los objetivos de este trabajo son estimar el coste económico de publicar en revistas de Psicología que ocupan posiciones de privilegio (cuartiles 1 y 2 en los JCR de 2017 y 2018), a través de las tasas de procesamiento de artículos y de publicación en abierto según la modalidad Golden OA o Green OA, y determinar si existen diferencias en el coste económico de publicación en función de diferentes variables como país de edición, editorial, cuartil y área temática de Psicología en las que se encuentran clasificadas las revistas en estas bases de datos.

\section{MÉTODO}

\subsection{Material y procedimiento}

Se volcaron en una base de datos todas las revistas con factor de impacto situadas en los cuartiles Q1 y Q2 que se encuentran recogidas en los JCR de los años 2017 y 2018, y que pertenecen, al menos, a una de las siguientes 11 categorías o áreas de la Psicología: "Psychology (SCIE)", y del SSCI las categorías "Psychology, Applied", "Psychology, Biological", "Psychology, Clinical", "Psychology, Developmental", "Psychology, Educational", "Psychology, Experimental", Psychology, Mathematical", "Psychology, Multidisciplinary", "Psychology, Social" y "Psychology, Psychoanalysis". 
La elección de la revistas de Psicología obedeció a diferentes razones, por un lado la Psicología ha sido una de las disciplinas que en mayor medida se ha servido de la bibliometría tras la Medicina (Delgado López-Cozar y otros 2006), por otro lado, las categorías temáticas de Psicología del JCR pueden ser más afines a las ciencias de la salud, como Psychology Clinical o Psychology Biological, y otras a las ciencias sociales, como Psychology Social, disyuntiva que se encuentra muchas veces cuando se evalúan los currículums de los investigadores en un área del conocimiento o en otra.

La variable Revistas fue definida por el número total de publicaciones diferentes incluidas en los JCR de 2017 y 2018, siendo 360 el número total de revistas analizadas. La variable número de registros hace referencia a las revistas incluidas en el año 2017 y a las incluidas en el 2018 de forma independiente. Se decidió construir esta variable por diferentes razones. Por un lado, de un año a otro podía variar el precio por publicar, teniendo en cuenta el valor del cambio de la moneda de dólares a euros y por otro lado, el número de trabajos publicados por una misma revista podía no ser el mismo de un año a otro.

El total de registros fue de 632 , de los cuales 317 pertenecen al JCR de 2017 y 315 al JCR de 2018. Del total de registros, 298 (47.2\%) están clasificadas en Q1 y 334 (52.8\%) en Q2. La distribución de los registros por país fue la siguiente: USA (391 registros, $61.9 \%)$, Inglaterra $(164,25.9 \%)$, Holanda $(38,6.0 \%)$, Alemania $(15,2.4 \%)$, España (11, $1.7 \%)$, Suiza $(7,1.1 \%)$, Australia $(2,0.3 \%)$ y con un solo registro Portugal, República Checa, Canadá y Bélgica. Cuando se analiza el área temática de la revista el número total de registros es de 763 , siendo este mayor al haber revistas que están clasificadas en más de una categoría dentro de la Psicología.

Los costes de publicación para cada revista se han tenido en cuenta a partir de la máxima visibilidad permitida desde la misma, fundamentalmente el coste desde Gold OA (Gold Open Access). De no existir esta posibilidad, se ha tenido en cuenta el coste de Green OA (Green Open Access) y de no ser así, los costes que la revista exige para la publicación de un artículo de corte científico en la misma, o APCs (Article Publication Charges). Todos estos costes eran consultados en la página de las revistas en el año 2019. En base a ello se tuvieron en cuenta dos variables, el coste por publicar un artículo en una revista y el coste total por revista, el cual se calculó multiplicando el número de trabajos publicados (artículos y revisiones) en los dos años analizados por el coste de publicar un artículo en la revista en ese año.
Por otro lado, se debe de tener en cuenta que las revistas de Psicología publicadas desde la American Psychological Association (APA), son gratuitas para los científicos dependientes de organizaciones gubernamentales, universidades o afiliaciones estadounidenses, pero no para cualquier otro considerado como extranjero, al cual se le solicita una cantidad económica por su publicación. En un principio se recogieron los costes en dólares y en euros, convirtiendo todos los costes a euros en función del cambio de la moneda a fecha 30 de junio de 2017 y 2018

Por último, se incluyó la variable "editorial" con las siguientes categorías: 1 . Universidades; 2 . Wiley; 3. Sage Publications; 4. American Psychological Association; 5. Elsevier; 6. Taylor \& Francis; 7. Springer; 8. Plenum Publishers; 8. Otras (la cual incluye colegios profesionales, fundaciones, sociedades y otros grupos editoriales diferentes a los mencionados en categorías anteriores, que se incluyen en esta categoría por la baja representatividad en la edición de revistas), y las variables factor de impacto y número de citas. En todos estos casos los costes totales estimados en función de estas variables se calcularon en base al número de registros y no al número de revistas.

\subsection{Análisis de datos.}

Se han realizado recuento de frecuencias y cálculos de medias. En los casos donde se han comparado las puntuaciones medias se han realizado pruebas $t$ de Student y ANOVA's con un nivel de significación de .05, realizándose correlaciones de Pearson en el estudio de la relación entre costes y factor de impacto y número de citas. Todos los análisis se realizaron mediante el paquete estadístico SPSS 24.

\section{RESULTADOS}

\subsection{Estimación de costes de publicación en las revistas de Psicología indexadas en el JCR de 2017 y 2018.}

La media de coste de publicación de un trabajo fue de 2321,55 euros (DT $=863,48$ ), siendo el coste mayor de 5352 euros en la revista Ergonomics.

Teniendo en cuenta los cargos por publicar en una revista y el número de trabajos publicados por año, la estimación del total económico que pueden llegar a pagar autores o instituciones por publicar en estas revistas, si lo hiciesen en acceso abierto, es de $135.514 .886,39$ euros, de los cuales $67.027 .213,11$ euros pertenecen a los trabajos publicados en las revistas del JCR en 2017, y $68.487 .673,28$ euros a los trabajos publicados en las revistas del JCR en 2018. 
Tabla I. Relación de revistas por año con mayor volumen económico estimado asociado a costes de publicación en euros.

\begin{tabular}{|l|l|r|}
\hline \multicolumn{1}{|c|}{ Año } & \multicolumn{1}{|c|}{ Revista } & \multicolumn{1}{c|}{ Costes en euros } \\
\hline \multirow{4}{*}{$\mathbf{2 0 1 7}$} & Frontiers in Psychology & $5.413 .489,32$ \\
\cline { 2 - 3 } & Frontiers in Human Neuroscience & $1.565 .564,55$ \\
\cline { 2 - 3 } & Personality and Individual Differences & $1.438 .857,2$ \\
\cline { 2 - 3 } & Computers in Human Behavior & $1.168 .285,16$ \\
\cline { 2 - 3 } & Journal of Autism and Developmental Disorders & 1.124 .864 \\
\hline \multirow{2}{*}{$\mathbf{2 0 1 8}$} & Frontiers in Psychology & $6.515 .422,2$ \\
\cline { 2 - 3 } & Frontiers in Human Neuroscience & $1.294 .437,9$ \\
\cline { 2 - 3 } & Psycho-Oncology & 1.254 .050 \\
\cline { 2 - 3 } & Journal of Autism and Developmental Disorders & 1.214 .720 \\
\cline { 2 - 3 } & Personality and Individual Differences & $1.053 .447,2$ \\
\hline
\end{tabular}

En la Tabla I se recogen las cinco revistas en las que el volumen económico por cargos es mayor, teniendo en cuenta el número de trabajos publicados en 2017 y en 2018. En ambos años son las revistas de la editorial Frontiers las que mayor volumen económico presentan por cargos por procesamiento de artículos.

De las 360 revistas analizadas, en 15 de ellas, lo que supone un $4.2 \%$ del total de revistas, no se cobran costes a los autores por publicar, mientras que en el $95.8 \%$ (345 revistas) sí se cobran cargos a los autores. En concreto, las revistas en las que no se cobran cargos son las siguientes: editadas en los Estados Unidos, Annual Review of Psychology, Annual Review of Clinical Psychology, Advances in Experimental Social Psychology, Annual Review of Organizational Psychology and Organizational Behavior, Judgement and Decision Making, Nonlinear Dynamics and Life Science, School Psychology Review y Bulletin of the Menninger Clinic. Editadas en Reino Unido, Behavioral and Brain Sciences. En
España, European Journal of Psychology Applied to Legal Context, Psicothema, Psicología Educativa, Revista de Psicodidáctica y Psychosocial Intervention. Y la editada en la República Checa, Cyberpsychology - Journal of Psychosocial Research on Cyberspace.

Cuando se analiza el coste de publicación por artículo, según el cuartil de las revistas (Q1 o Q2), no existen diferencias estadísticamente significativas $t(596,520)=-.035, p=.972$, no habiendo diferencias tampoco en cuanto al coste total de los trabajos publicados según el cuartil $\mathrm{t}(627)=-.463, \mathrm{p}=643$.

Con respecto a la editorial existen diferencias estadísticamente significativas en relación a los costes medios por publicar $F_{(8,630)}=48.088 ; p=.000$ habiendo solamente 3 editoriales en las que se puede encontrar alguna revista donde no se tengan que pagar cargos por publicar (ver Tabla II). El coste medio por publicar un artículo es mayor en las revistas editadas por la American Psychological Association (APA), mientras que el coste medio menor se da en la editorial Springer.

Tabla II. Costes de publicación en euros según la editorial de la revista.

\begin{tabular}{|l|c|c|c|c|c|c|}
\hline Editorial & Reg. & Media (DT)* & Mínimo* & Máximo* & Media (DT)** & Máximo** \\
\hline Universidades & 27 & $2025,69(1072,34)$ & 0 & 3146 & $235468,97(179303,74)$ & 642240 \\
\hline Wiley & 113 & $2489,83(374,64)$ & 1795,68 & 3583 & $187805,70(182270,12)$ & 1254050 \\
\hline Sage Publications & 74 & $2550,71(316,56)$ & 877,19 & 3448,27 & $163632,86(163.632,86)$ & 646550 \\
\hline APA & 80 & $3439,16(214,64)$ & 2275 & 3508,77 & $247557,69(247.557,69)$ & 641378,22 \\
\hline Elsevier & 116 & $1868,96(601,98)$ & 0 & 3333,33 & $260881,80(260.881,80)$ & 1438857,20 \\
\hline Taylor \& Francis & 99 & $2287,61(601,96)$ & 494 & 5352 & $131916,72(132261,53)$ & 765336 \\
\hline Springer & 39 & $1405,42(710,13)$ & 305 & 2356 & $110942,46(110.942,46)$ & 285076 \\
\hline Plenum Publishers & 20 & $2467,44(953,82)$ & 641 & 3498 & $365528,08(365.528,08)$ & 1214720 \\
\hline Otros & 63 & $1865,62(1391,33)$ & 0 & 4544 & $340225,28(340.225,28)$ & 6515422,20 \\
\hline
\end{tabular}

DT (Desviación Típica). *Descriptivos en relación al coste medio en euros por publicar un artículo en revistas de la editorial. ** Descriptivos en relación al coste total por publicar en revistas de la editorial. 
Cuando se analiza el coste total por publicar teniendo en cuenta la editorial, de nuevo aparecen diferencias estadísticamente significativas $\mathrm{F}_{(8,628)}=2.731 ; \mathrm{p}=$ .006. En este caso el coste medio total mayor corresponde a las revistas de la editorial Plenum Publishers, mientras que las editoriales con mayor volumen económico total son las de las revistas pertenecientes a la categoría de Otros y a la editorial Elsevier.

Cuando se analizan los costes que se han de pagar por publicar en función del país de edición de la revista, se puede observar que solamente hay un país en el que no se cobran cargos, la República Checa, si bien sólo cuenta con una publicación. En cambio, son las revistas editadas en Alemania en primera instancia y en Suiza, las que presentan costes medios mayores. Estos resultados pueden observarse en la Tabla III.
Con respecto a las áreas temáticas de Psicología, es el área de "Psychology Mathematical" donde los costes medios por publicar un artículo son menores, seguida por la categoría "Psychology Educational". Por su parte, es en las categorías de "Psychology Developmental" y "Psychology (SCIE)" donde mayor es el coste medio por publicar un trabajo. En todas las áreas se puede encontrar algún registro (referente a revistas) donde no se cobran cargos por publicar, salvo en el área de "Psychology Developmental". Todos estos datos pueden consultarse en la Tabla IV. Estas diferencias resultaron ser estadísticamente significativas $\mathrm{F}_{(10,761)}=4.300, \mathrm{p}=.000$.

En relación a los costes totales, se puede observar (ver Tabla V) cómo el coste medio mayor corresponde a las publicaciones del área "Psychology Biological" en primer lugar y "Psychology (SCIE)", mientras

Tabla III. Costes de publicación en euros por país de edición de la revista.

\begin{tabular}{|l|r|r|r|r|r|}
\hline País & No. registros & Media & $\begin{array}{c}\text { Desviación } \\
\text { Típica }\end{array}$ & \multicolumn{1}{c|}{ Mínimo } & Máximo \\
\hline EEUU & 391 & 2424,26 & 864,59 & 0.0 & 3596,49 \\
\hline Inglaterra & 164 & 2278,63 & 691,39 & 0.0 & 5352 \\
\hline Holanda & 38 & 2131,26 & 492,57 & 1018 & 2931,03 \\
\hline Suiza & 7 & 2611,80 & 66,49 & 2543,1 & 2723 \\
\hline Alemania & 14 & 2738,49 & 1091,29 & 1551,72 & 4544 \\
\hline España & 11 & 181,81 & 404,51 & 0 & 1000 \\
\hline Portugal & 1 & 305 & & 305 & 305 \\
\hline Australia & 2 & 2083 & & 2083 & 2083 \\
\hline Rep. Checa & 1 & 0 & & 0 & 0 \\
\hline Canadá & 1 & 2586,20 & & 2586.20 & 2586.20 \\
\hline Bélgica & 1 & 450 & & 450 & 450 \\
\hline
\end{tabular}

Tabla IV. Costes de publicación en euros según el área temática de Psicología en el JCR 2017 y 2018.

\begin{tabular}{|l|c|c|c|r|r|}
\hline \multicolumn{1}{|c|}{ Áreas temáticas } & No. registros & Media & $\begin{array}{c}\text { Desviación } \\
\text { Típica }\end{array}$ & Mínimo & Máximo \\
\hline Applied & 85 & 2314,01 & 923,44 & 0 & 5352 \\
\hline Biological & 15 & 2231,29 & 994,92 & 0 & 3508,77 \\
\hline Clinical & 134 & 2442,10 & 806,97 & 0 & 4148,6 \\
\hline Developmental & 77 & 2515,35 & 662,36 & 437 & 3508,77 \\
\hline Educational & 56 & 1987,66 & 949,19 & 0 & 3508,77 \\
\hline Experimental & 93 & 2251,06 & 875,5 & 0 & 4544 \\
\hline Mathematical & 13 & 1121,04 & 869,71 & 0 & 2631,57 \\
\hline Multidisciplinary & 136 & 2286,75 & 940,8 & 0 & 4148,6 \\
\hline Psychoanalysis & 11 & 2475,00 & 1417,95 & 0 & 4148,6 \\
\hline Social & 64 & 2433,69 & 779,73 & 0 & 3508,77 \\
\hline Psychology (SCIE) & 78 & 2509,77 & 1063,81 & 0 & 5352 \\
\hline Total & 762 & 2330,78 & 912,72 & 0 & 5352 \\
\hline
\end{tabular}


Tabla V. Costes totales en euros por publicación de trabajos según las diferentes áreas temáticas de Psicología (JCR 2017 y 2018).

\begin{tabular}{|l|c|c|c|c|}
\hline Área temáticas & No. registros & Media & Desviación Típica & Máximo \\
\hline Applied & 85 & 155208.47 & 169149,32 & 830788 \\
\hline Biological & 15 & 323469,58 & 286474,89 & 984210 \\
\hline Clinical & 134 & 215532,64 & 162838,39 & 825515 \\
\hline Developmental & 77 & 261734,06 & 222983,76 & 1214720 \\
\hline Educational & 56 & 109983,69 & 105765,15 & 547250 \\
\hline Experimental & 93 & 237040,88 & 201551,53 & 1168285 \\
\hline Mathematical & 13 & 74607 & 48146,12 & 131896 \\
\hline Multidisciplinary & 136 & 250044,01 & 729476,57 & 6515422 \\
\hline Psychoanalysis & 11 & 94518,42 & 59113,74 & 168421 \\
\hline Social & 64 & 187876,95 & 226837,47 & 1438857 \\
\hline Psychology (SCIE) & 78 & 317035,23 & 293941,09 & 1565565 \\
\hline Total & 762 & 220540,68 & 362021,19 & 6515422 \\
\hline
\end{tabular}

Tabla VI. Distribución de registros sin costes y con costes de publicación por áreas de Psicología.

\begin{tabular}{|l|c|c|c|}
\hline Área temática & $\mathbf{N}^{\mathbf{0}} \mathbf{y}$ \% de registros sin costes & $\mathbf{N} \mathbf{.} \mathbf{y} \%$ de registros con costes & Total \\
\hline Applied & $3(3.5)$ & $82(96.5)$ & 85 \\
\hline Biological & $2(13.3)$ & $13(86.7)$ & 15 \\
\hline Clinical & $4(3.0)$ & $130(97.0)$ & 134 \\
\hline Developmental & $0(0.0)$ & $77(100)$ & 77 \\
\hline Educational & $3(5.4)$ & $53(94.6)$ & 56 \\
\hline Experimental & $2(2.2)$ & $91(97.8)$ & 93 \\
\hline Mathematical & $1(7.7)$ & $12(92.3)$ & 13 \\
\hline Multidisciplinary & $12(8.8)$ & $124(91.2)$ & 136 \\
\hline Psychoanalysis & $1(8.3)$ & $11(91.7)$ & 12 \\
\hline Social & $2(3.1)$ & $62(96.9)$ & 64 \\
\hline Psychology (SCIE) & $6(7.7)$ & $72(92.3)$ & 78 \\
\hline Total & $36(4.7)$ & $727(95.3)$ & 763 \\
\hline
\end{tabular}

que el coste medio menor se produce en las áreas "Psychology Mathematical" y "Psychology Psychoanalysis". Estas diferencias resultaron ser estadísticamente significativas $\mathrm{F}_{(10,761)}=2.114, \mathrm{p}=.021$.

En relación al área que presenta unos costes totales mayores, es el área de "Psychology Multidisciplinary", lo cual es comprensible si se tiene en cuenta que es la que presenta un mayor número de registros, mientras que la categoría de "Psychology Mathematical" es la que recoge el menor número de costes totales.

Cuando se analizan los registros en relación a si pertenecen a revistas con costes o sin costes por publicar en función de las diferentes áreas temáticas en las que se encuentran clasificadas las revistas de Psicología (ver Tabla VI), es la categoría de "Psycho- logy Biological" la que cuenta con un mayor número de registros publicados en revistas sin costes de publicación, con un $13.3 \%$. Mientras tanto, en la categoría "Psychology Developmental" todos los trabajos publicados presentan cargos por publicar.

Por último, se relacionaron los costes por publicar un artículo y los costes totales con dos indicadores bibliométricos, como son el factor de impacto y el número total de citas que recibe la revista. Los análisis muestran que no existe relación entre los costes por artículo $(p=.205)$ y totales $(p=.181)$ con el factor de impacto, en cambio, existe una relación negativa estadísticamente significativa entre el coste por publicar en una revista $(R x y=-.115, p$ $=.004)$ y los costes totales ( $R x y=-.123, p=.002)$ con el número de citas. 


\section{DISCUSIÓN Y CONCLUSIONES.}

La accesibilidad a los resultados científicos a través de los artículos y revisiones publicados en revistas científicas de acceso abierto ha llevado, en la mayoría de las ocasiones, un coste para los propios autores e instituciones (Kaufman, 2014), aspecto que también se puede constatar en las revistas de Psicología analizadas en el presente estudio, ya que aproximadamente en el $95 \%$ de las revistas se han de pagar unas tasas por procesamiento de artículos y/o publicación en Open Access (OA), situando este cargo en unos $2321,55 €$ por trabajo, cantidad ciertamente notable, que además fluctuará en función de la demanda percibida por la revista y sus criterios editoriales específicos.

Cuando se analizan en conjunto los costes estimados de publicación en estas revistas durante los dos años analizados, se puede comprobar el considerable volumen económico que supone la publicación científica en abierto, aproximadamente unos 135.515.000 euros. Estas cantidades ingentes, llevan a reflexionar acerca de si la comunicación de resultados científicos se ha convertido en un negocio, donde los principales beneficiarios son las editoriales, y en concreto los grandes grupos como Elsevier (Holanda), Springer (USA), Wiley-Blackwell (USA) Taylor-Francis (Gran Bretaña) y Sage (USA), que como apuntan Larivière, Haustein y Mongeon (2015) tienen el oligopolio de las revistas indizadas en la Web of Science al estar detrás del $70 \%$ de las publicaciones incluidas en el Social Sciences Citation Index. Si bien, son las revistas de la APA las que tienen unos costes por publicar en abierto mayores, cargos que no son cobrados a los autores de instituciones norteamericanas, son las editoriales Elsevier y Wiley las que sumando el número de revistas y de trabajos publicados presentarían unos ingresos por estos conceptos mayores.

Tal y como apuntan Melero y Abad (2008) la publicación en abierto permite considerar los resultados científicos como un bien común, al ser compartidos tanto con la comunidad científica y profesionales como con la sociedad en general. Pero, también el pagar por publicar presenta inconvenientes, principalmente en relación a aquellas áreas o temas científicos que no cuentan con tanta demanda, por lo que pueden disponer de menos financiación, o en relación a los jóvenes investigadores que tienen más limitado su entrada en proyectos de investigación con financiación (Swan y Brown, 2004). Teniendo en cuenta los costes medios por publicar y la cantidad de dinero que pueden llegar a pagarse por cargos de procesamiento de artículos en las revistas de Psicología situadas en los dos primeros cuartiles del JCR de 2017 y 2018, la reflexión que la comunidad científica ha de hacer y que ya ha sido apuntada en otros trabajos en relación a las revistas de medicina (López-Torres, 2015), es cuánto un autor está dispuesto a pagar y cuál sería un precio razonable en relación al procesamiento de artículos que tendrían que cobrar las revistas, teniendo en cuenta que muchas de estas revistas si bien han reducido costes al publicar online, también al estar en JCR pueden haber incrementado el número de trabajos que reciben y por ello presentar más costos relacionados con el procesamiento de artículos que son rechazados y no generan ingresos. Además, unas tasas mayores por este concepto no garantizan un mayor número de citas si se tiene en cuenta la relación negativa existente entre costes y número de citas, resultado que entra en contradicción con el estudio de Dewatripont y otros (2006).

También, debemos recordar que pese a los costes por publicar que exigen las editoriales, los revisores de las revistas científicas no suelen cobrar por su labor de análisis y revisión de los artículos enviados, por lo que los beneficios de las editoriales se acumulan únicamente para gestión de las mismas. Se sabe que los revisores son científicos prestigiosos de las disciplinas donde desarrollan su trabajo, así que, por un lado deben de realizar una labor de revisión gratuita, y por otro, pagarse los costes de publicación desde la, a veces, escasa financiación recibida desde los organismos públicos a los que suelen adscribirse.

Las revistas científicas han ocupado un espacio relevante, casi exclusivo, en la transmisión de los resultados científicos frente a los libros y otras tipologías documentales, permitiendo el consumo de la información científica entre investigadores. Pero en el escenario económico y de evaluación del profesorado universitario, donde uno de los criterios relevantes es el número de citas que reciben los trabajos publicados por un investigador ( $\sin$ que se entienda excesivamente bien en qué sentido la calidad docente puede ser "medida" por la vía alternativa y harto diferente de la calidad investigadora), cabe preguntarse qué precio se paga por ser citado. En este sentido, González-Sala y otros (2019) encuentran que en las revistas iberoamericanas de Psicología, entre los años 2012 y 2016, entre un $88.8 \%$ y un $55.8 \%$ de las citas que reciben los trabajos son autocitas de autor principalmente, o de la propia revista, por lo que son los propios autores, así como sus grupos de referencia, los principales consumidores y difusores de los trabajos que producen. Llegados a este punto, la reflexión que cabría realizar es si, en determinadas ocasiones y contextos, los autores no están pagando por citarse a sí mismos. 
Pero, para entender el entramado desde la perspectiva de la sociología de la ciencia, hay que tener en cuenta otra variable relevante, y es la necesidad de publicar en revistas de excelencia, considerándose como tales aquellas que están clasificadas en los primeros cuartiles del JCR. Esta necesidad ha de crearse, si bien, como apunta Merton (1942) desde la perspectiva del ethos científico, cualquier revista con rigor metodológico en la evaluación de los trabajos que publica, como bien pudiera ser la revisión anónima por pares, podría ser un medio óptimo para publicar los resultados científicos sean estos cuales fueren. Por otro lado, como apuntan Osca-Lluch y otros (2019) esta necesidad de publicar en revistas de excelencia, pone en peligro la perdurabilidad y sostenimiento de otras revistas no indexadas entre los primeros cuartiles, que ciertamente no cobran por publicar y serían una vía de difusión científica especializada tan valiosa como cualquier otra, pero que, simplemente, tienen problemas para recibir una cantidad razonable de trabajos de calidad.

Numerosos estudios señalan la gran proyección que tienen los artículos que se encuentran simplemente disponibles online, libres para su descarga, además de conseguir éstos incrementar su nivel de citación gracias a dicha disponibilidad (Ale Ebrahim y otros, 2014). Todos estos factores sugieren la necesidad de repensar la dirección hacia la que se encamina actualmente el conocimiento científico y la gestión económica de su publicación.

En el caso de España, la necesidad de publicar en revistas de impacto, entendiendo éstas como aquellas que están indexadas en los dos primeros cuartiles del JCR de la WoS, se entiende si se tienen en cuenta los criterios para la obtención de sexenios y de acreditación para la carrera docente e investigadora. De hecho, es el factor más relevante del proceso. Ello lleva a los investigadores a intentar publicar en estas revistas como primera opción, y teniendo en cuenta que sólo un $4.2 \%$ de las revistas de Psicología editadas en los JCR 2017 y 2018 no llevan asociados gastos para los autores, la reflexión es de nuevo la misma: qué precio se paga por conseguir los criterios estipulados por las Agencias de Evaluación de la Calidad Científica (Agencia Nacional de Evaluación y Prospectiva -ANEP-, Comisión Nacional Evaluadora de la Actividad Investigadora -CNEAI- y la Agencia Nacional de Evaluación de la Calidad y Acreditación -ANECA-) para la consecución de los méritos de cara a la acreditación de la carrera docente e investigadora $y$, en qué medida la forma de evaluar las carreras científicas contribuye a seguir con el negocio de la publicación científica. Es decir, la obtención de un currículum se convierte en una inversión consistente en publicar en revistas de "impacto", y muy posiblemente dirigiendo buena parte del dinero que se destina a los proyectos de investigación, que solo pueden obtenerse por vía curricular, en financiar a empresas editoriales extranjeras. Tal necesidad ha llevado a la tentación de falsificar el currículum y los resultados de las investigaciones, pudiéndose hablar de un "trastorno patológico" de la propia ciencia (Buela-Casal, 2014).

Otro aspecto a destacar es que las tasas por procesamiento de artículos y/o publicación en abierto dependen de diferentes variables. En este sentido cabe destacar el caso de las revistas editadas en España, y en concreto cinco publicaciones, European Journal of Psychology Applied to Legal Context, Psicothema, Psicología Educativa, Revista de Psicodidáctica y Psychosocial Intervention, así como en la revista editada en la República Checa Cyberpsychology - Journal of Psychosocial Research on Cyberspace, en las que no se cobran cargos por publicar. En el caso de las revistas españolas, cuatro de ellas, son editadas o coeditadas por el Colegio Oficial de Psicólogos de España (Tortosa-Pérez y otros, 2020) en lo que supone un claro ejemplo de que existen otras estrategias y alternativas de gestión de las revistas que no supongan un coste masivo, y a veces inasumible, para los propios autores.

Otra de las variables estudiadas que determina las tasas medias de pago ha sido el área temática de la Psicología en la que se encuentra clasificada la revista, destacando como son las categorías de "Psychology, Mathematical" y "Psychology, Educational" las que presentan un menor coste para los autores. Estas diferencias entre revistas de una misma área científica pueden relacionarse con las halladas por Björk y Solomon (2015) entre revistas biomédicas y revistas de humanidades y sociales, si se tiene en cuenta que hay revistas de Psicología más afines a las ciencias de la salud y otras más relacionas con las ciencias sociales y las humanidades. En este sentido, en futuros estudios cabría dilucidar qué variables pueden estar condicionando estas diferencias en función del área temática, como bien pudiera ser el nivel de innovación investigadora, y el peso que juegan determinados indicadores bibliométricos, como el número de trabajos publicados financiados por proyectos de investigación, el número de revistas en la categoría, el número de firmantes por trabajo o aspectos como el número de descargas de artículos o la tasa de rechazo de las revistas.

La principal limitación del presente estudio se relaciona con los materiales seleccionados, al contem- 
plarse registros únicamente de dos años y pertenecientes a los dos primeros cuartiles del JCR de la Web of Science. Futuros estudios han de ir dirigidos a analizar los costes de publicación en otras bases de datos y en revistas de diferentes cuartiles.

\section{REFERENCIAS}

Ale Ebrahim, N.; Salehi, H.; Embi, M.; Tanha, F.; Gholizadeh, H. ; y Motahar, S. M. (2014). Visibility and Citation Impact. International Education Studies, 7(4), 120125. https://doi.org/10.5539/ies.v7n4p120

Barschall, H. (1988). The Cost-effectiveness of physics journals. Physics Today, July 1988, 56-59. Disponible en: http://www.library.yale.edu/barschall/articles/ pt8807.pdf [Fecha de consulta: febrero 2020]. https:// doi.org/10.1063/1.881125

Barrueco, J. M. (2010). El acceso abierto: amenaza u oportunidad. Revista Española de Drogodependencias, 35(2), 116-119. Disponible en: http://www.aesed. com/descargas/revistas/v35n2_ed.pdf [Fecha de consulta: diciembre 2019].

Bergstrom, T. (2001). Free labour for costly journals? Journal of Economic Perspectives, 15 (4), 183-198. https://doi.org/10.1257/jep.15.4.183

Björk, B.-C.; y Solomon, D. (2015). Article processing charges in OA journals: relationship between price and quality. Scientometrics, 103(2), 373-385. https://doi. org/10.1007/s11192-015-1556-z

Buela-Casal, G. (2014). Pathological publishing: A new psychological disorder with legal consequences? The European Journal of Psychology Applied to Legal Context, 6(2), 91-97. http://dx.doi.org/10.1016/j. ejpal.2014.06.005

Buela-Casal, G.; Bermúdez, M. P.; Sierra, J. C.; Quevedo-Blasco, R.; Guillén-Riquelme, A.; y Castro, A. (2013). Productividad y eficiencia en la investigación por Comunidades Autónomas españolas según la financiación (2011). Aula Abierta, 41(2), 87-98.

Buela-Casal, G.; Bermúdez, M. P.; Sierra, J. C.; Quevedo-Blasco, R.; Guillén-Riquelme, A.; y Castro, A. (2012). Productividad y eficiencia en la investigación de 2010: relación con la financiación de las Comunidades Autónomas españolas. Revista Electrónica de Metodología Aplicada, 17(1), 35-50

Claudio-González, M. G.; Martín-Baranera, M.; Villaroya Planas, A. (2017). La edición de revistas científicas en España: una aproximación descriptiva. Anales de Documentación, 20(1), 1-13. https://doi.org/10.6018/ analesdoc.20.1.265771

Dallmeier-Thiessen, S.; Darby, R.; Goerner, B.; Hyppoelae, J.; Igo-Kemenes, P.; Kahn, D.; Lambert, S.; y van der Stelt, W. (2011). Open Access publishing today: What scientist do and why. 2011 SOAP Symposium. Berlin. http://www.slideshare.net/ProjectSoap/ soap-symposiumtalkiii

Delgado López-Cózar, E. (2018a). Sexenios 2018, cambios relevantes: reformando la evaluación de la transferencia de conocimiento y con novedades del Tribunal Supremo. 4a Ed. Granada, 17 de diciembre de 2018. Disponible en: http://hdl.handle.net/10481/54307 [Fecha de consulta: febrero 2020].
Delgado López-Cózar, E. (2018b). De la ruta de oro a la ruta verde de la comunicación científica: negocio editorial y bibliométrico, publicación libre, acceso abierto, evaluación total e individualizada. http://dx.doi. org/10.13140/RG.2.2.30852.78724

Delgado López-Cózar, E.; y Cordón García, J. A. (1991). Le transfert de l'information scientifique et technique: le role des Nouvelles technologies de l'information face à la crise du modèle actuel de communication écrite. Revue de Bibliologie: Schèmas et Schèmatissations, 34, 78-85.

Delgado López-Cózar, E.; Torres-Salinas, D.; JiménezContreras, E.; y Ruiz-Pérez, R. (2006). Análisis bibliométrico y de redes sociales aplicado a las tesis bibliométricas defendidas en España (1976-2002): temas, escuelas científicas y redes académicas. Revista Española de Documentación Científica, 29(4), 493524. http://dx.doi.org/10.3989/redc.2006.v29.i4

Dewatripont, M. y col. (2006). Study on the economic and technical evolution of the scientific publication markets in Europe, Final Report, European Commission, Directorate General for Research. Disponible en: https://op.europa.eu/en/publication-detail/-/publication/1058c2f8-5006-4d13-ae3f-acc6484623b9 [Fecha de consulta: enero 2020].

González-Sala, F.; Osca-Lluch, J.; y Haba-Osca, J. (2019). Are journal and author self-citations a visibility strategy? Scientometrics, 119(8), 1345-1364. https://doi. org/10.1007/s11192-019-03101-3

Hernández Borges, A. A. ; Cabrera Rodríguez R.; Montesdeoca Melián, A.; Martínez Pineda, B.; Torres Álvarez de Arcaya, M. L.; y Jiménez Sosa, A. (2006). Awareness and attitude of Spanish medical authors to OA publishing and the "author pays" model. Journal of the Medical Library Association, 94(4), 449-451.

Kaufman R. (2014). Shifting revenues from post-publication to pre-publication: The impact of Open Access. ALPSP Blog. Disponible en: http://blog.alpsp.org/2014/03/ roy-kaufman-shifting-revenues-from-post.html [Fecha de consulta: febrero 2020].

Laakso, M.; y Björk, B. C. (2012). Anatomy of Open Access publishing-A study of longitudinal development and internal structure. BMC Medicine, 10(124). https://doi.org/10.1186/1741-7015-10-124

Larivière, V.; Haustein, S.; y Mongeon, P. (2015). The oligopoly of academic publishers in the digital era. PLoS ONE, 10, e0127502. https://doi.org/10.1371/journal. pone. 0127502

López-Torres Hidalgo, J. (2015). "Pagar por publicar" en revistas científicas. Revista Clínica de Medicina de Familia, 8(3), 179-181. http://dx.doi.org/10.4321/ S1699-695X2015000300001

Martín González, J. C.; y Merlo Vega, J. A. (2003). Las revistas electrónicas: características, fuentes de información y medios de acceso. Anales de Documentación, 6, 155-186. https://www.researchgate.net/publication/279475177_Las_revistas_electronicas_caracteristicas_fuentes_de_informacion_y_medios_de_acceso. https://doi.org/10.6018/analesdoc.6.0.1971

Melero, R.; y Abad, M. F. (2008). Revistas open access: características, modelos económicos y tendencias. Textos Universitaris de Biblioteconomia i Documentació, 20. Disponible en: http://bid.ub.edu/20meler2. htm. [Fecha de consulta: enero 2020]. 
Merton, R. K. (1942). The normative structure of science [excerpts] (originally published as "Science and Technology in a Democratic Order"). Panarchy: a gateway to selected documents and web sites. Disponible en https://www.panarchy.org/merton/science.html. [Fecha de consulta: enero 2020].

Ministerio de Ciencia, Innovación y Universidades (2018). Resolución de 14 de noviembre de 2018, de la Comisión Nacional Evaluadora de la Actividad Investigadora, por la que se publican los criterios específicos aprobados para cada uno de los campos de evaluación. BOE, 26 de noviembre de 2018.

Musi-Lechuga, B.; Olivas-Ávila, J. A.; Portillo-Reyes, V.; t Villalobos-Galvis, F. (2005). Producción de los profesores funcionarios de Psicología en España en artículos de revistas con factor de impacto de la Web of Science. Psicothema, 17(4), 539-548.

Nassi-Calò, L. (2013) ¿Cuánto cuesta publicar en acceso abierto? [online]. SciELO en Perspectiva. Disponible en: https://blog.scielo.org/es/2013/09/18/ cuanto-cuesta-publicar-en-acceso-abierto/ [Fecha de consulta: abril 2020].

Osca-Lluch, J.; González-Sala, F.; Haba-Osca, J.; Tortosa, F.; y Peñaranda-Ortega, M. (2019). Comunicación científica o cualificación para una Carrera académica: ¿Qué uso tienen los artículos en las revistas de psicología? Anales de Psicología, 35(1), 166-174. http://dx.doi.org/10.6018/analesps.35.1.329211
Osuna, E. (2009). Calidad y financiación de la universidad. Revista de Investigación en Educación, 6, 133-141. Disponible en http://reined.webs.uvigo.es/index.php/reined/article/view/60 [Fecha de consulta: enero 2020].

Swan, A.; y Brown, S. (2004). Authors and OA publishing. Learned Publishing, 17(3), 219-224. https://doi. org/10.1087/095315104323159649

Tortosa-Pérez, M.; González-Sala, F.; Santolaya-Prego de Oliver, J.; y Aguilar-Bustamante, C. (2020). El papel de la organización colegial-COP en el posicionamiento internacional de la Psicología Española (19792018). Anales de Psicología, 36(1), 12-23. https://doi. org/10.6018/analesps.388691

Walters, W. H. (2014). Do article influence scores overestimate the citation impact of social science journals in subfields that are related to higher-impact natural science disciplines? Journal of Informetrics, 8(2), 421430. https://doi.org/10.1016/j.joi.2014.02.001

Wiley (2013). Wiley Open Access author survey 2013, Presentation material. Disponible en: http://www. slideshare.net/WileyScienceNewsroom/wileys-2013open-access-author-survey [Fecha de consulta: enero 2020].

Zhao, D. (2010). Characteristics and impact of grant-funded research: a case study of the library and information science field. Scientometrics, 84(2), 293-306. https://doi.org/10.1007/s11192-010-0191-y 